\title{
Remarks on Long Range Interactions in Simulations
}

\author{
E.L. Pollock
}

This paper was prepared for submittal to the Workshop on Treatment of Electrostatic Interactions in Computer Simulations of Condensed Media

Santa Fe, NM

June 23-25, 1999

August 11, 1999

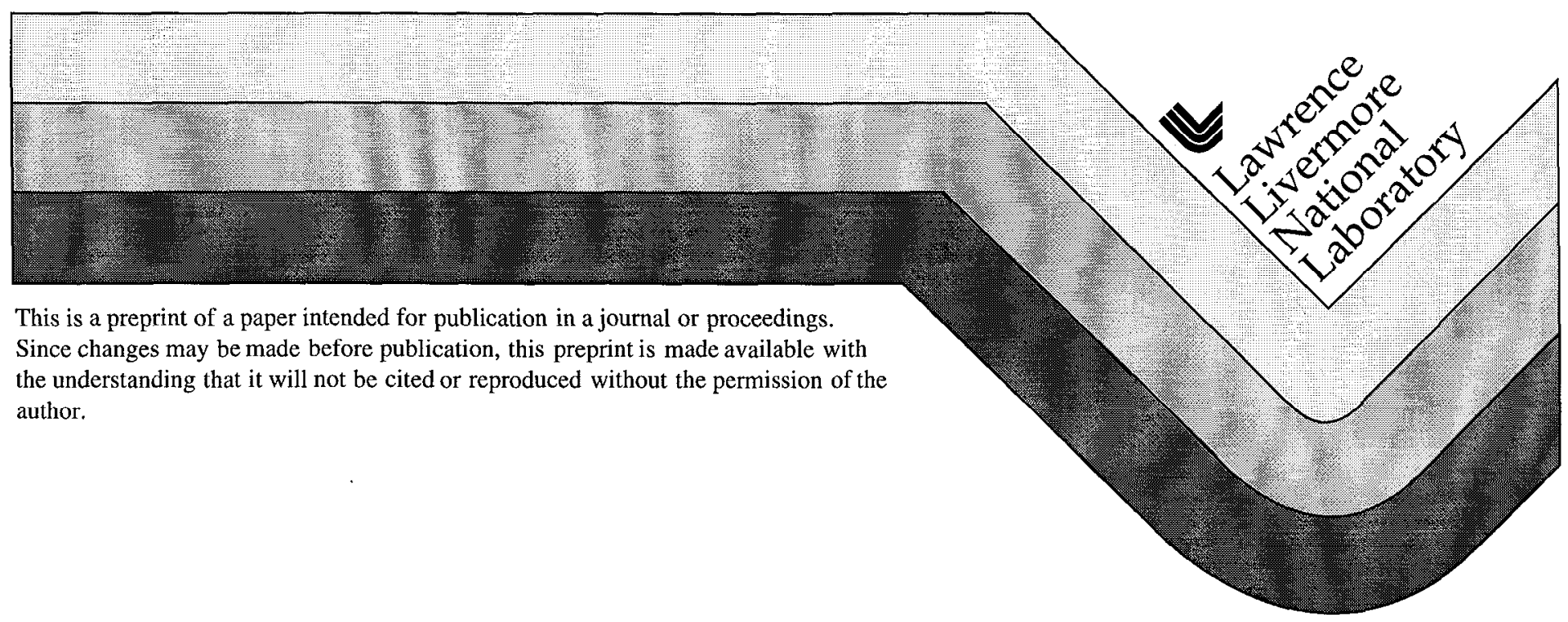




\section{DISCLAIMER}

This document was prepared as an account of work sponsored by an agency of the United States Government. Neither the United States Government nor the University of California nor any of their employees, makes any warranty, express or implied, or assumes any legal liability or responsibility for the accuracy, completeness, or usefulness of any information, apparatus, product, or process disclosed, or represents that its use would not infringe privately owned rights. Reference herein to any specific commercial products, process, or service by trade name, trademark, manufacturer, or otherwise, does not necessarily constitute or imply its endorsement, recommendation, or favoring by the United States Government or the University of California. The views and opinions of authors expressed herein do not necessarily state or reflect those of the United States Government or the University of California, and shall not be used for advertising or product endorsement purposes. 


\title{
Remarks on Long Range Interactions in Simulations
}

\author{
E. L. Pollock \\ Physics Department \\ Lawrence Livermore National Laboratory \\ Livermore, CA 94550
}

\begin{abstract}
Long range interactions in periodic systems should be treated using the Ewald potential. For systems larger than a few hundred particles this is best calculated using the particle-particle, particle-mesh $\left(\mathrm{P}^{3} \mathrm{M}\right)$ method.
\end{abstract}

\section{INTRODUCTION}

Although most of the simulations I've worked on involve the Coulomb potential, since along with the Fermi exclusion principle it accounts for most of the properties of condensed matter systems, two distinct problems stand out where a proper treatment and understanding of the long range nalure of the interaction was the focus. This talk recounts in a colloquial fashion the lessons drawn from these two problems.

The first, done in collaboration with B. J. Alder almost twenty years ago, concerned the calculation of the dielectric properties of polar condensed matter systems. An understanding of how to apply fluctuation relations or external field methods allowed the first, to my knowledge, correct computation of the dielectric properties, including frequency [1] and wave vector dependence [2] and polarizability [3] of a polar liquid.

The second was part of a recent collaboration involving Livermore and Sandia National Labs, Bristol Meyers Squibb, Dupont, and the then Cray Research to develop a massively parallel code to simulate biomolecular or polymeric systems. 'I'his code is now available free under licensing agreement [4]. In the course of this we concluded that the $\mathrm{P}^{3} \mathrm{M}$ method developed by Hockney and Eastwood [5], as opposed to a direct Ewald or fast multipole method was the most efficient way of treating the long range interactions in large $(N \geq 200$ particles $)$ on either single or multiprocessor machines. 


\section{EWALD POTENTIALS, BOUNDARY CONDITIONS, TRUNCATION EFFECTS}

The Coulomb potential energy sum, $U$, in an extended system is conditionally convergent. What is needed to detine it is a summation order, typically given physically by considering a system of a certain shape (e.g. sphere, slab, etc.) which is maintained while the system expands. The result is [6]

$$
U=U^{\text {Ewald }}+\text { shape dependent stuff. }
$$

Most textbook derivations of the Ewald potential, where an absolutely convergent sum mysteriously appears after the application of two nonobvious integral identities, ignore this question. [7]

For a generalized ellipsoidal shaped crystal the electrostatic potential per periodic cell,

$$
U=U^{E w a l d}+\frac{1}{2 \Omega}\left(A P_{x}^{2}+B P_{y}^{2}+C P_{z}^{2}\right)
$$

where the shape dependent factors $A+B+C=4 \pi$ and $\mathbf{P}$ is the dipole moment per periodic cell of volume $\Omega$ The Ewald potential thus does not contain the shape dependent surface terms.

The most detailed derivation of this result is that of deLeeuw, Perram, and Smith [8]. There are also several simpler intuitive ways of seeing this.

A slight generalization of the usual Ewald derivation can be applied to the screened Coulomb potential, $\exp (-r / \lambda) / r$. At the end of the calculation the zero wave vector terms vanish because of charge neutrality and the screening length can then be taken arbitrarily large to get the usual E'wald form which suggests that boundary effects are not included.

As a more direct example consider the local field in a cubic lattice of unit dipoles oriented along the $\hat{z}$ axis. The dipolar lattice sum can be exactly summed over a large spherical region and the remaining sum done as an integral which is converted after integration by parts to a surface integral over the inner spherical surface $\left(\partial \Omega_{1}\right)$ and the outer surface of the crystal $\left(\partial \Omega_{2}\right)$

$$
\sum_{\mathbf{L}}\left(\frac{3 L_{z}^{2}}{L^{5}}-\frac{1}{L^{3}}\right)=\sum_{\text {sphere }}\left(\frac{3 L_{z}^{2}}{L^{5}}-\frac{1}{L^{3}}\right)-\int_{\partial \Omega} \frac{z}{r^{3}} d A_{z}
$$

where $\partial \Omega=\partial \Omega_{1}+\partial \Omega_{2}$. The sum over the sphere is zero by cubic symmetry and the inner surface integral evaluates to $-4 \pi / 3$ so the local field expression reduces to

$$
\sum_{\mathbf{L}}\left(\frac{3 L_{z}^{2}}{L^{5}}-\frac{1}{L^{3}}\right)=\frac{4 \pi}{3}-\int_{\partial \Omega_{2}} \frac{z}{r^{3}} d A_{z}
$$


The outer surface integral can be done for simple cases, giving for example $4 \pi / 3$ again for a spherical sample and thus a local field of 0 . For a slab perpendicular to the $\hat{z}$ axis the integral is $4 \pi$ and the resulting local field $-8 \pi / 3$.

What happens if the local field sum is evaluated by the Ewald formula? The result is $4 \pi / 3$ showing that the outer surface integral is not included.

It is apparent from Eq. 2 that boundaries suppress polarization fluctuations. A surface polarization produces a field which resists further polarization. If, as in the calculation of dielectric properties, the result is to be obtained from polarization fluctuations [9] then their suppression is clearly undesirable. The Ewald potential corresponds to the best boundary condition for this type of calculation.

To expand on these remarks: the dielectric constant is defined by the relation between the average polarization density and the Maxwell electric field

$$
\frac{<\mathrm{P}>}{\Omega}=\frac{(\epsilon-1)}{4 \pi}<\mathrm{E}>\text {. }
$$

A non-zero average polarization can be produced by an external field, or in terms of the Hamiltonian

$$
H=H_{0}-\mathbf{P} \cdot \mathbf{E}_{e x t}
$$

so

$$
e^{-\beta H}=e^{-\beta H_{0}}\left(1+\beta \mathbf{P} \cdot \mathbf{E}_{e x t}+O\left(\mathbf{E}_{e x t}^{2}\right)\right) .
$$

This gives the linear response result

$$
<\mathrm{P}>=\beta<\mathrm{PP}>\cdot \mathrm{E}_{e x t}
$$

and by comparison with the definition

$$
\frac{(\epsilon-1)}{4 \pi}<\mathbf{E}>=\beta \Omega<\mathbf{P P}>\cdot \mathbf{E}_{e x t} .
$$

Since the dielectric properties are independent of the sample shape, the polarization fluctuations will mirror the relation between the Maxwell and the external field.

For example in the case of a slab perpendicular to $\hat{z}, E_{z}=E_{z}^{\text {ext }} / \epsilon$ and from the preceding equation

$$
4 \pi \beta \Omega<P_{z}^{2}>=(1-1 / \epsilon) .
$$

The polarizations fluctuations perpendicular to the slab face saturate as $\epsilon$ increases and it would be impossible to accurately determine a large $\epsilon$ from these fluctuations in a simulation of the system. This is also true for a truncated interaction. "Reaction field" methods have been shown, after the fact, to be workable but they seem unnecessary, typically require several iterations to check for size dependence. and violate the spirit of periodic boundary conditions. 
For the Ewald potential $\langle\mathbf{E}\rangle=\mathbf{E}_{\text {ext }}$ so

$$
4 \pi \beta \Omega<\mathbf{P}^{2}>/ 3=(\epsilon-1) .
$$

The polarization fluctuations scale linearly with $\epsilon$ which can now be accurately computed.

Although examples can be and were given at this conference of large macromolecules where the interest is in events in a small region and it might be desirable to replace most of the system with a bath or dielectric continuum, in general the remark about socialism attributed to Oscar Wilde applies to dielectric continuum: "It takes up too many evenings". Discussions about where to start the continuum approximation (2nd shell, 3rd shell of neighbors etc.), whether to treat it as responding linearly or nonlinearly to the central discrete part of the system, what other properties to include seem never to cease and it's better they never start. Application of Moore's law, the doubling of computer power every two years or so, is perhaps the preferred solution.

\section{$\mathbf{P}^{3} \mathrm{M}$}

To follow the advice given in the last paragraph it is necessary to find an efficient way to evaluate Coulomb interactions for large systems. We advocate and review the particle-particle, particle-mesh method discussed in the book of Hockney and Eastwood [5] and several recent articles [10], [11]. Our remarks are condensed from reference [12]. The $\mathrm{P}^{3} \mathrm{M}$ method can be routinely applied to any Ewald formulation (inverse power interactions, dipolar interactions, vortex-vortex interactions etc.) but we will focus on the Coulomb case.

The $\mathrm{P}^{3} \mathrm{M}$ method will be introduced by a sequence of three conceptual steps the first two of which are displayed in the cartoon below. We first repeat a standard, non-rigorous, derivation for the Coulomb Ewald formula by adding and subtracting Gaussian charge densities from the point charges to get the usual short ranged and smooth long ranged terms. In step two the Gaussian densities forming the smooth long ranged term, evaluated in Fourier space, are then discretized in order to use FFT techniques. In the third step these Gaussian densities are replaced by a simpler grid assignment function and the Coulomb Green's function correspondingly modified to give the final $\mathrm{P}^{3} \mathrm{M}$ formulation.

As shown by the first arrow in the cartoon below, Gaussian charge densilies are added and subtracted from the original point charges in a periodic cell.

The Coulomb sum

$$
\begin{aligned}
U=\sum_{i} \sum_{<j} \sum_{\mathbf{L}} \frac{Q_{i} Q_{j}}{\left|\mathbf{r}_{i j}+\mathbf{L}\right|} & \Rightarrow \sum_{i} \sum_{<j} \sum_{\mathbf{L}}\left[\frac{Q_{i} Q_{j}}{\left|\mathbf{r}_{i j}+\mathbf{L}\right|}-\iint \frac{\rho_{i}(\mathbf{r}) \rho_{j+L}\left(\mathbf{r}^{\prime}\right)}{\left|\mathbf{r}+\mathbf{r}^{\prime}\right|} d \mathbf{r} d \mathbf{r}^{\prime}\right] \\
& +\sum_{i} \sum_{<j} \sum_{\mathbf{L}} \iint \frac{\rho_{i}(\mathbf{r}) \rho_{j+L}\left(\mathbf{r}^{\prime}\right)}{\left|\mathbf{r}+\mathbf{r}^{\prime}\right|} d \mathbf{r} d \mathbf{r}^{\prime}
\end{aligned}
$$




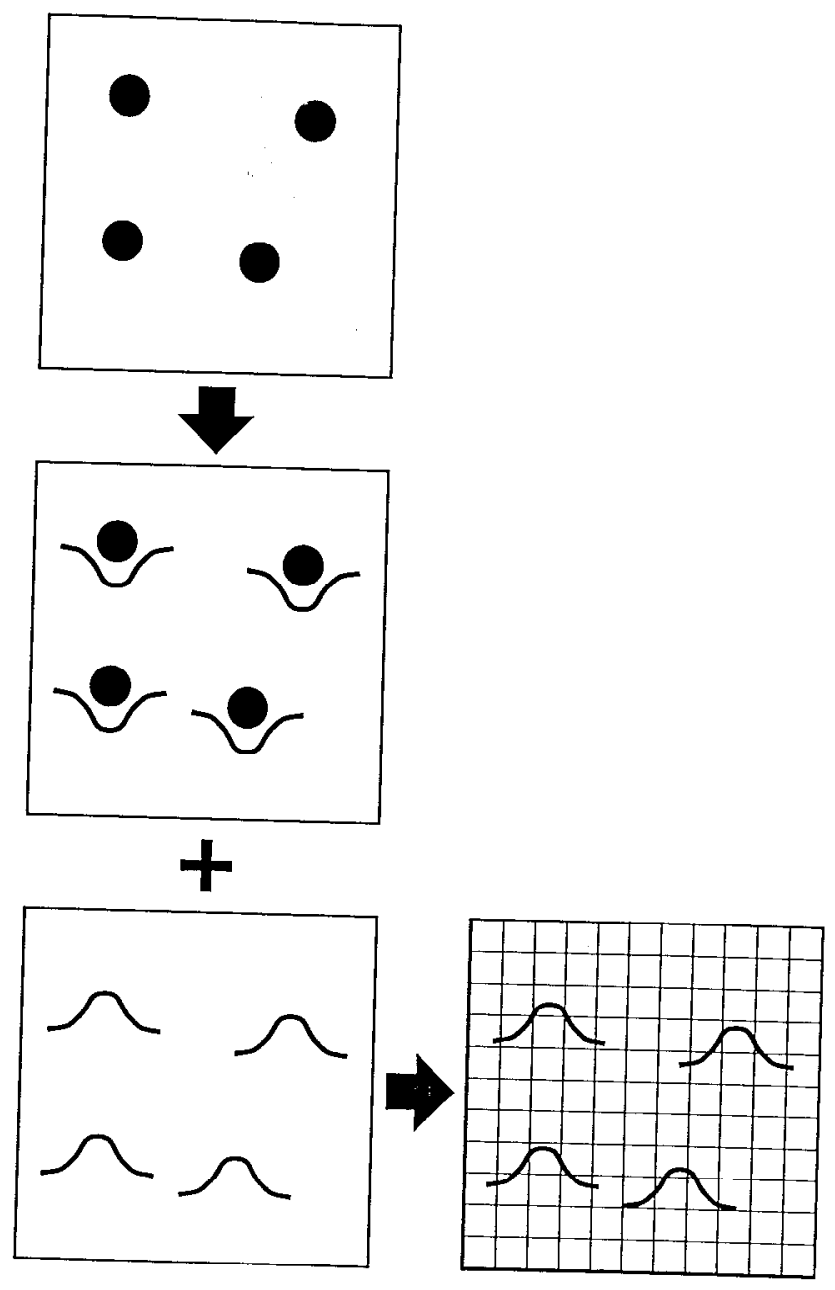

FIGURE 1. Cartoon showing first two steps in explaining the $P^{3} \mathrm{M}$ method: addition and subtraction of Gaussian densities to, here, four charges in the periodic cell to derive the usual Ewald formula, and the imposition of a grid to derive the mesh Ewald method

where the Gaussian charge density

$$
\rho_{i}(\mathbf{r})=Q_{i}\left(\frac{G^{2}}{\pi}\right)^{3 / 2} e^{-G^{2}\left(r-\mathbf{r}^{\prime}\right)^{2}}
$$

The first, short ranged, term is evaluated analytically and the second term transformed to Fourier space to get the usual Ewald formula

$$
U=\sum_{i} \sum_{<j} \sum_{\mathbf{L}} \frac{Q_{i} Q_{j} \operatorname{erfc}\left(G\left|\mathbf{r}_{i j}+\mathbf{L}\right| / \sqrt{2}\right)}{\left|\mathbf{r}_{i j}+\mathbf{L}\right|}+\sum_{\mathbf{k} \neq 0} \frac{4 \pi}{L^{3} k^{2}} e^{-k^{2} / 2 G^{2}}|S(\mathbf{k})|^{2}-\frac{G}{\sqrt{2 \pi}} \sum_{i} Q_{i}^{2}
$$

with $S(\mathbf{k})=\sum_{j=1}^{N} Q_{j} e^{i \mathbf{k} \cdot \mathbf{r}_{j}}$ and $\mathbf{k}=\frac{2 \pi}{L} \mathbf{n}, n=0 \ldots \infty$. 
For $\mathrm{N}$ charges in the periodic cell the time to evaluate the two terms is

$$
T=c_{1} N \frac{\rho}{G^{3}}+c_{2} N \frac{G^{3}}{\rho} N
$$

where $c_{1}$ and $c_{2}$ are constants. When this is minimized with $G, G^{3} \sim \rho / \sqrt{N}$ and the familiar $T \sim N^{3 / 2}$ scaling results. If $G$ is fixed the scaling degrades to $O\left(N^{2}\right)$.

In the second step, sometimes called mesh Ewald, a grid is imposed and the Gaussian densities are discretizcd so

$$
\sum_{\mathbf{k} \neq 0} \frac{4 \pi}{L^{3} k^{2}} e^{-k^{2} / 2 G^{2}}|S(\mathbf{q})|^{2} \Rightarrow \sum_{\mathbf{q} \neq 0} \frac{4 \pi}{L^{3} q^{2}}|\rho(\mathbf{q})|^{2}
$$

where $\mathbf{q}=\frac{2 \pi}{L} \mathbf{n}, n=0 \ldots M-1$ with the grid spacing $\Delta=L / M$ and $\rho(\mathbf{q})$ is the discrete Fourier transform of the total Gaussian density.

The accuracy of this discretization depends on the Gaussian width divided by the grid spacing, $\sim 1 / G \Delta$, so for a given accuracy $\Delta \sim 1 / G$ and the number of grid points $M^{3}=L^{3} / \Delta^{3} \sim N G^{3} / \rho$. With a discrelized density the FFT can be used. The time necessary to evaluate $U$ is now

$$
T=c_{1} N \frac{\rho}{G^{3}}+c_{2} N+c_{3} \frac{N G^{3}}{\rho} \ln \left(\frac{N G^{3}}{\rho}\right)+c_{4} \frac{N G^{3}}{\rho} .
$$

The successive terms are for evaluating the short range terms, as before; the time to form $\rho(\mathbf{r})$ on the grid;the time necessary to calculate the $\rho(\mathbf{q})$; and finally the time required to do the sum. Minimizing with $G$ now leads to $G^{3} \sim 1 / \sqrt{\ln N}$ and ' $T$ ' $\sim \sqrt{\ln N}$. Now a non-optimal $G$ degrades the scaling only to $N \ln N$. This is important in practice since the range of possibly other short range interactions may dictate the choice of $G$.

The third step of replacing the Gaussian densities by a finitc rangc assignment function gives the final form of the $\mathrm{P}^{3} \mathrm{M}$ method. The simplest assignment function, $W_{1}$, assigns the charge density to the nearest grid point in each direction. The full assignment function being a product of $W$ s along each axis. The higher order assignment functions are convolutions of $W_{1}, W_{n}=W_{1} * W_{n-1}$, and span $\mathrm{n}$ grid points in each direction. The first four such functions are shown below (solid lines) in figure 2 and by the law of large numbers quickly converge back to Gaussians (dashed line).

Successive increases in $n$ increase the accuracy of the force calculation by roughly an order of magnitude as shown in figure 3. The use of a finite range assignment function speeds up the calculation but does not change the basic scaling with number of particles. It is also advantageous for parallel processing codes using a domain decomposition technique since only particles within a thin layer of $n / 2$ grid points from an adjacent domain contribute to the density on that domain thus reducing the necessary message passing. 


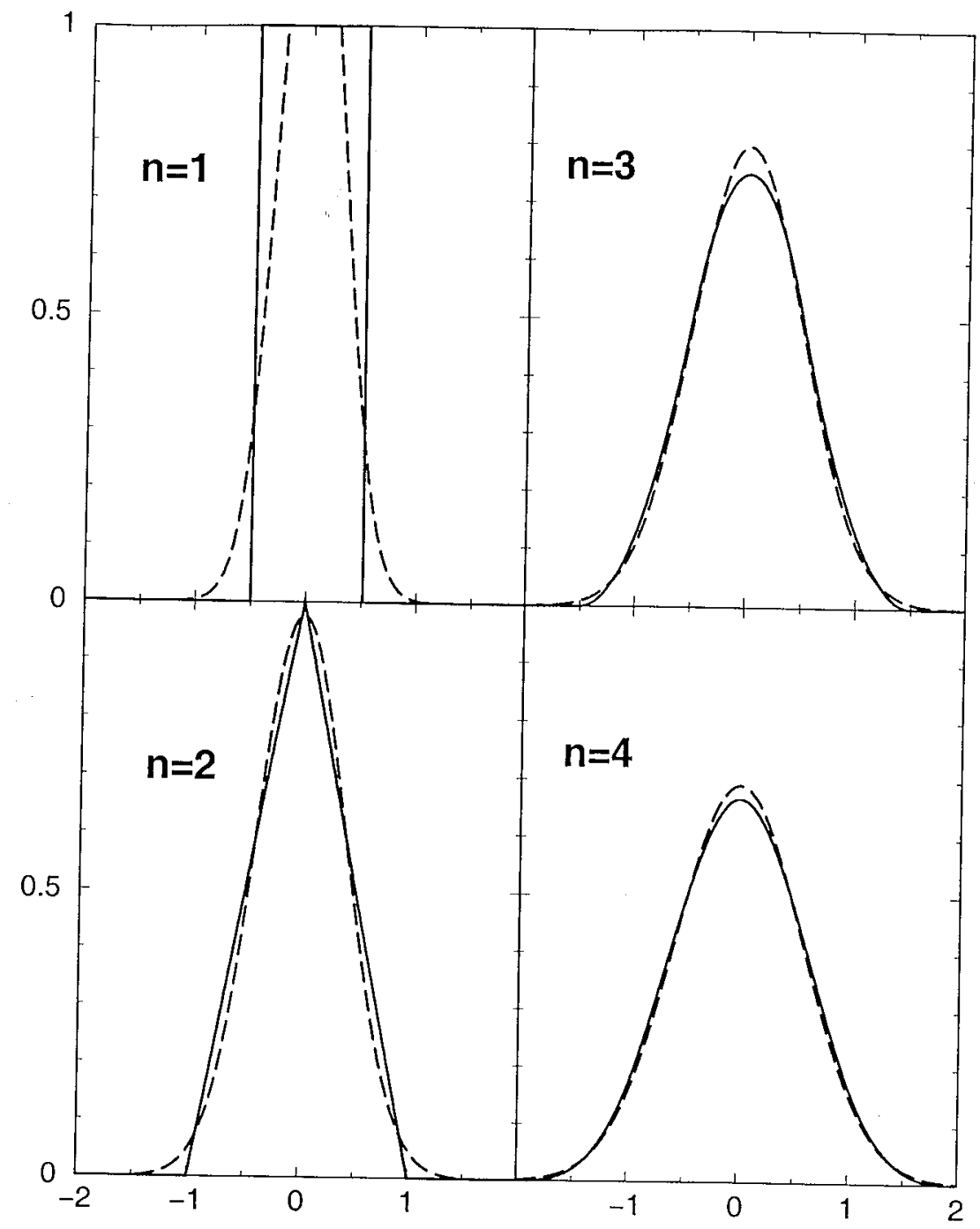

FIGURE 2. First 4 grid assignment functions (solid line) and approximating Gaussians (dashed line), $\sigma^{2}=n / 12$.

Since the $\rho(\mathbf{q})$ of Eq. 17 have now been replaced by $W_{n}(\mathbf{q})$ the Coulomb Green's function, $4 \pi / \mathbf{q}^{2}$, is replaced by $T_{n}(\mathbf{q})$. A derivation of $T_{n}(\mathbf{q})$ is given in the appendix. For large $n$ and large grids it converges again to $4 \pi / \mathbf{q}^{2}$.

Recounting, the steps involved in a $\mathrm{P}^{3} \mathrm{M}$ calculation: the charge density of the $N$ particles is assigned to the grid

$$
\rho\left(\mathbf{r}_{g}\right)=\sum_{j=1}^{N} Q_{j} W_{n}\left(\mathbf{r}_{g}-\mathbf{r}_{j}\right)
$$

this density is Fourier transformed using an FFT, $\rho\left(\mathbf{r}_{g}\right) \Rightarrow \rho(\mathbf{q})$; the inverse transform of $i \mathbf{q} T_{n}(\mathbf{q}) \rho(\mathbf{q})$ gives the electric field at the grid points, $\mathbf{E}\left(\mathbf{r}_{g}\right)$; and finally the electric field is interpolated back to the particles,

$$
\mathbf{E}\left(\mathbf{r}_{j}\right)=\sum_{g} W_{n}\left(\mathbf{r}_{j}-\mathbf{r}_{g}\right) \mathbf{E}\left(\mathbf{r}_{g}\right)
$$




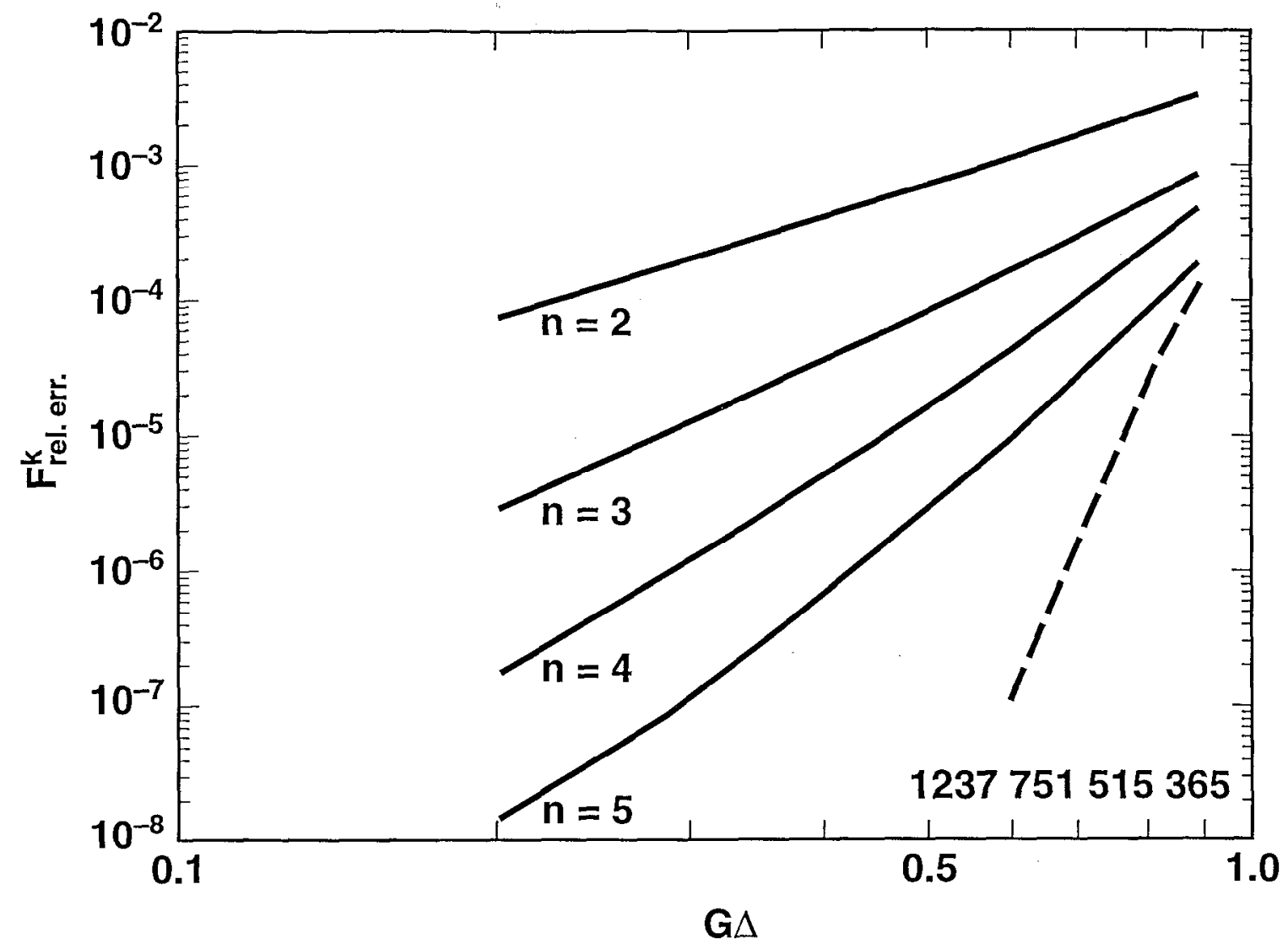

FIGURE 3. Relative accuracies of the Fourier space part of the forces for various choices of grid assignment function. 'The dashed curve is for the mesh Ewald method and the numbers above the $\mathrm{x}$-axis give the number of grid points around each Gaussian that must be included with this method. For $\mathrm{P}^{3} \mathrm{M}$ each charge is assigned to $n^{3}$ grid points.

As Hockney and Eastwood show the same $W_{n}$ must be used in assigning the charge to the grid and in interpolating the field back to the particles in order to satisfy Newton's third law.

Figure 4 compares typical times for a force calculation on a single processor machine for three methods; standard Ewald, $\mathrm{P}^{3} \mathrm{M}$, and the fast multipole method FMM, as a function of the number of particles in the periodic cell. As discussed in more detail in reference $[12] \mathrm{P}^{3} \mathrm{M}$ has several advantages over $F M M$. From personal experience and reports in the literature a casually written FMM code can be ponderously slow even if scaling as $O(N)$. The FMM code, written by Jim Glosli, used in fig. 4 was highly optimized as indicated by the low crossover between the FMM and standard Ewald timings.

Since much of the impetus to develop fast methods came from simulations of 


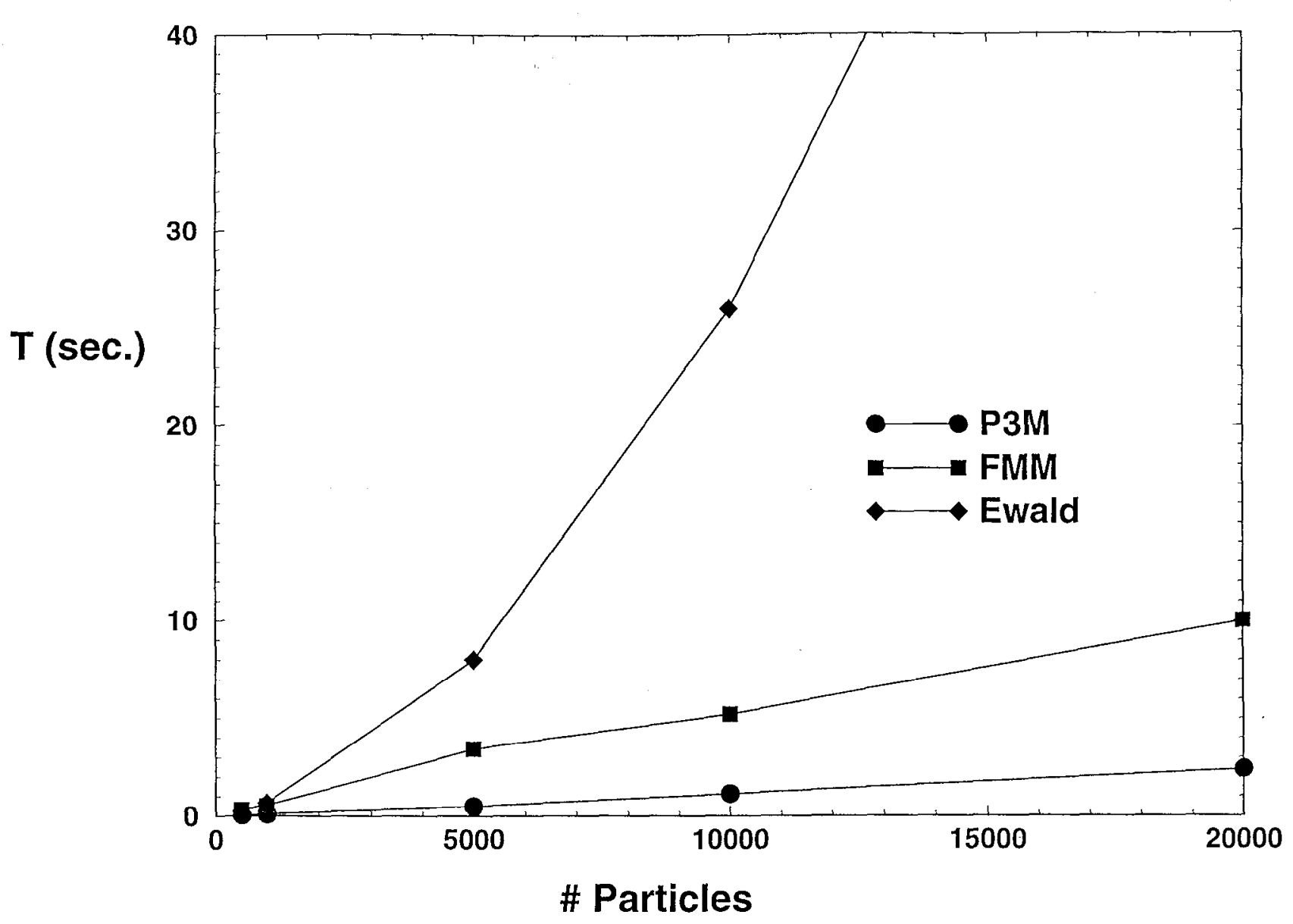

FIGURE 4. Timings for complete force calculation of various size systems using standard Ewald, fast multipole method, and $\mathrm{P}^{3} \mathrm{M}$. Details are given in reference [12].

nonperiodic systems primarily gravitational interactions in star clusters, as a final topic we discuss how finite geometries such as spherical clusters or slabs are handled with $\mathrm{P}^{3} \mathrm{M}$. The idea is the same for both. Since the speed of the method comes from the FFT we wish to maintain a periodic system. This is done by truncating the interaction (either in 3 or 1 dimensions here) such that all intcractions in the original system are unchanged but the fictitious periodic images do not interact which will require an appropriate periodic cell size.

For example in a finite cluster contained within a bounding sphere of radius $R$, taking the interaction truncation radius $R_{c} \geq 2 R$ leaves all interactions in the cluster unchanged. Taking the periodic coll length $L \geq R_{c}+2 R$ assures that periodic images don't interact.

Repeating the derivation of Eq. 15 for this truncated potential the short range (erfc) terms are unaffected, as long as $R_{c}$ exceeds the overlap of the Gaussians which is trivially achieved for a large system. In the second, Fourier term, the Fourier 
transform of the truncated potential, i.e. $\left(4 \pi / k^{2}\right)\left[1-\cos \left(k R_{c}\right)\right]$ replaces $4 \pi / k^{2}$. The transition to $\mathrm{P}^{3} \mathrm{M}$ proceeds exactly as before. For a non-neutral or gravitational cluster the $k=0$ term must be included which adds $\Delta U=\left(\pi R_{c}^{2} / L^{3}\right) \sum_{j} Q_{j}^{2}$ to the potential.

The case of a periodic slab in the $x-y$ plane proceeds similarly with $4 \pi / k^{2}$ now replaced by

$$
\frac{4 \pi}{k^{2}}\left\{\begin{array}{l}
{\left[1-e^{-k_{\|} Z_{c}}\left(\cos \left(k_{z} Z_{c}\right)-\frac{k_{z}}{k_{\|}} \sin \left(k_{z} Z_{c}\right)\right)\right]} \\
{\left[1-\cos \left(k_{z} Z_{c}\right)\right] \text { for } k_{\|}=0}
\end{array}\right.
$$

where $k_{\|}=\sqrt{k_{x}^{2}+k_{y}^{2}}$.

The use of a larger periodic cell and thus larger number of grid points only affects the FFTs which are typically $10 \%$ or less of the overall calculation so the final timings are not seriously increased.

A discussion of the multi-processor aspects of $\mathrm{P}^{3} \mathrm{M}$ can be found in ref. [12]. The bulk of the computational lime is spent in assigning the charge density to the grid and in interpolating the field from the grid to the charged particles. The time for these operations scales inversely with the number of processors. The multiprocessor FFT, which as stated above takes roughly $10 \%$ of the computational time, does not inherently scale inversely with the number of processors. It is difficult to make a general statement but typically for an $M^{3}$ grid deviations of the FFT from inverse scaling are about $10 \%$ at $M$ processors and increases for larger numbers of processors. So far in our experience this has not been a serious, or even mild, limitation on the use of $\mathrm{P}^{3} \mathrm{M}$ on multiprocessor computers.

\section{A OPTIMAL $T_{N}$}

The optimal $T_{n}(\mathbf{q})$ is chosen by minimizing the mean squared difference between the electric field at $r$ produced by a charge at $r^{\prime}$ calculated exactly from the Ewald formula and by the $\mathrm{P}^{3} \mathrm{M}$ approximation for arbitrary choice of $r$ and $r^{\prime}$ in the periodic cell. The short range terms are the same for Ewald and $\mathrm{P}^{3} \mathrm{M}$ so only the particle-mesh, Fourier space, terms are considered. Repeating, the quantity

$$
Q \equiv \int d \mathbf{r} \int d \mathbf{r}^{\prime}\left[\mathbf{E}^{P M}\left(\mathbf{r} ; \mathbf{r}^{\prime}\right)-\mathbf{E}^{E w}\left(\mathbf{r} ; \mathbf{r}^{\prime}\right)\right]^{2}
$$

is to be minimized with respect to $T_{n}(\mathbf{q})$.

We will use $\mathrm{k}$ with $k_{x}=(2 \pi / L)[0, \ldots, \infty]$ for the full Fourier series and $\mathbf{q}$ with $q_{x}=(2 \pi / L)[0, \ldots, M-1]$ for the discrete version for functions tabulated on a grid of $M^{3}$ points. The difference between the full and discrete series

$$
\sum_{\mathrm{k}}=\sum_{\mathrm{q}} \sum_{\mathrm{b}}
$$

is the inclusion of the Brillouin zone vectors $\mathbf{b}$ with $b_{x}=(2 \pi / \Delta)[0, \ldots, \infty]$ and $\Delta=L / M$, or the appropriate generalization for a non cubic periodic cell. 
In the Ewald formula the Fourier space part of the "electric field"

$$
\mathbf{E}^{E w}\left(\mathbf{r} ; \mathbf{r}^{\prime}\right)=\sum_{\mathbf{k} \neq 0} i \mathbf{k} T(k) e^{-i \mathbf{k} \cdot\left(\mathbf{r}-\mathbf{r}^{\prime}\right)} .
$$

For the Coulomb case

$$
T(k)=\frac{4 \pi}{L^{3} k^{2}} e^{-k^{2} / 2 G^{2}}
$$

but we shall keep it general to allow for other long range interactions.

The explicit expression for $\mathbf{E}^{P M}\left(\mathbf{r} ; \mathbf{r}^{\prime}\right)$ is somewhat more complicated but follows straight forwardly from the steps outlined in the preceding section,

$$
\mathbf{E}^{P M}\left(\mathbf{r} ; \mathbf{r}^{\prime}\right)=\sum_{\mathbf{g}} W_{n}(\mathbf{r}-\mathbf{g}) \mathbf{E}_{\mathbf{g}}
$$

with

$$
\mathbf{E}_{\mathbf{g}}=\sum_{\mathbf{q}} i \mathbf{q} T_{n}(\mathbf{q}) W_{n}(\mathbf{q}) e^{i \mathbf{q} \cdot \mathbf{r}_{\mathbf{g}}}
$$

and

$$
W_{n}(\mathbf{q})=\frac{1}{M^{3}} \sum_{\mathbf{g}^{\prime}} W_{n}\left(\mathbf{r}^{\prime}-\mathbf{g}^{\prime}\right) e^{-i \mathbf{q} \cdot \mathbf{r}^{\prime} \mathbf{g}^{\prime}}
$$

This gives

$$
\mathbf{E}^{P M}\left(\mathbf{r} ; \mathbf{r}^{\prime}\right)=\frac{1}{M^{3}} \sum_{\mathbf{g}} \sum_{\mathbf{g}^{\prime}} W_{n}(\mathbf{r}-\mathbf{g})\left[\sum_{\mathbf{q}} i \mathbf{q} T_{n}(\mathbf{q}) e^{-i \mathbf{q} \cdot\left(\mathbf{r}_{\mathbf{g}}-\mathbf{r}_{\mathbf{g}^{\prime}}\right)}\right] W_{n}\left(\mathbf{r}^{\prime}-\mathbf{g}^{\prime}\right)
$$

To do the integrals over the periodic cell in the definition of $Q$ the Fourier transform

$$
W_{n}(\mathbf{r})=\sum_{\mathbf{k}} W_{n}(\mathbf{k}) e^{-i \mathbf{k} \cdot \mathbf{r}}
$$

is needed. The full series over $\mathbf{k}$ is used since the $W_{n}(\mathbf{r})$ are analytic functions and not limited to values on a grid. The identity

$$
\sum_{\mathbf{g}} e^{i(\mathbf{k}-\mathbf{q}) \cdot \mathbf{r}_{\mathbf{g}}}=M^{3} \delta_{\mathbf{k}, \mathbf{q}+\mathbf{b}}
$$

allows the sums over $\mathbf{g}$ and $\mathbf{g}^{\prime}$ to be done giving

$$
\mathbf{E}^{P M}\left(\mathbf{r} ; \mathbf{r}^{\prime}\right)=M^{3} \sum_{\mathbf{q}} i \mathbf{q} T_{n}(\mathbf{q}) e^{-i \mathbf{q} \cdot\left(\mathbf{r}-\mathbf{r}^{\prime}\right)} \sum_{\mathbf{b}} W_{n}(\mathbf{q}+\mathbf{b}) e^{-i \mathbf{b} \cdot \mathbf{r}} \sum_{\mathbf{b}^{\prime}} W_{n}\left(\mathbf{q}+\mathbf{b}^{\prime}\right) e^{-i \mathbf{b}^{\prime} \cdot \mathbf{r}^{\prime}}
$$

The terms in $Q$ may now be evaluated using 


$$
\int d \mathbf{r} e^{i \mathbf{k} \cdot \mathbf{r}}=L^{3} \delta_{\mathbf{k}, 0}
$$

and also noting that $\delta_{\mathbf{q}+\mathbf{b}, \mathbf{q}^{\prime}+\mathbf{b}^{\prime}}=\delta_{\mathbf{q}, \mathbf{q}^{\prime}} \delta_{\mathbf{b}, \mathbf{b}^{\prime}}$.

$$
\begin{gathered}
\int d \mathbf{r} \int d \mathbf{r}^{\prime} F^{E w}\left(\mathbf{r} ; \mathbf{r}^{\prime}\right) \cdot F^{E w}\left(\mathbf{r} ; \mathbf{r}^{\prime}\right)=L^{6} \sum_{\mathbf{k} \neq 0} k^{2} T^{2}(k) \\
\int d \mathbf{r} \int d \mathbf{r}^{\prime} F^{E w}\left(\mathbf{r} ; \mathbf{r}^{\prime}\right) \cdot F^{P M}\left(\mathbf{r} ; \mathbf{r}^{\prime}\right)=M^{3} L^{6} \sum_{\mathbf{q}} \sum_{\mathbf{b}}(\mathbf{q}+\mathbf{b}) \cdot \mathbf{q} T(\mathbf{q}+\mathbf{b}) T_{n}(\mathbf{q}) W_{n}^{2}(\mathbf{q}+\mathbf{b}) \\
\int d \mathbf{r} \int d \mathbf{r}^{\prime} F^{P M}\left(\mathbf{r} ; \mathbf{r}^{\prime}\right) \cdot F^{P M}\left(\mathbf{r} ; \mathbf{r}^{\prime}\right)=M^{3} L^{6} \sum_{\mathbf{q}} \sum_{\mathbf{b}} \sum_{\mathbf{b}^{\prime}} q^{2} T_{n}^{2}(\mathbf{q}) W_{n}^{2}(\mathbf{q}+\mathbf{b}) W_{n}^{2}\left(\mathbf{q}+\mathbf{b}^{\prime}\right)
\end{gathered}
$$

Using these and varying $Q$ with $T_{n}(\mathbf{q})$ gives the optimal

$$
T_{n}(\mathbf{q})=\frac{1}{M^{3}} \frac{\sum_{\mathbf{b}} \frac{\mathbf{q} \cdot(\mathbf{q}+\mathbf{b})}{q^{2}} T(\mathbf{q}+\mathbf{b}) W_{n}^{2}(\mathbf{q}+\mathbf{b})}{\left[\sum_{\mathbf{b}} W_{n}^{2}(\mathbf{q}+\mathbf{b})\right]^{2}} .
$$

The denominator can be summed exactly and the numerator is quickly convergent. The $T_{n}(\mathbf{q})$ are calculated once at the beginning of the simulation. If the simulation involves varying the periodic cell then, of course, it is necessary to recalculate for each new periodic cell.

\section{ACKNOWLEDGMENTS}

This work was performed under the auspices of the U.S. Department of Energy by Lawrence Livermore National Laboratory under contract No. W-7405-Eng-48.

\section{REFERENCES}

1. Pollock, E. L., and Alder, B. J., Phys. Rev. Lett. 46, 950 (1981).

2. Pollock, E. L., and Alder, B. J., Physica 102A, 1 (1980), Physica 105 A, 363 (1981).

3. Pollock, E. L., Alder, B. J., and Patey, G. N., Physica 108A, 14 (1981).

4. For information or license agreement contact Steve Plimpton, sjplimp@cs.sandia.gov

5. Hockney, R. W., and Eastwood, J. W., Computer Simulation Using Particles, Adam Hilger (1988)

6. Redlack, A., Grindlay, J., J. Phys. Chem. Solids 36,73 (1975).

7. Ziman J. M., Principles of the Theory of Solids Cambridge University Press (1965) 
8. DeLeeuw, S. W., Perram, J. W., and Smith, E. R., Proc. R. Soc. Lond. A373,27 (1980).

9. Felderhof, B. U.,Physica 101A,275 (1980).

10. Luty, B. A., Davis, M. E., Tironi, I. G., VanGunsteren, W. F.,Mol. Sim. 14,11 (1994).

11. Darden, T. A., York D., Pederson, L. J. Chem. Phys. 98, 10089 (1993) and subsequent work.

12. Pollock, E. L., and Glosli, J., Comp. Phys. Comm. 95, 93 (1996) 